\title{
GFVO: the Genomic Feature and Variation Ontology
}

Joachim Baran, Bibi Sehnaaz Begum Durgahee, Karen Eilbeck, Erick Antezana, Robert Hoehndorf, Michel Dumontier

Falling costs in genomic laboratory experiments have led to a steady increase of genomic feature and variation data. Multiple genomic data formats exist for sharing these data, and whilst they are similar, they are addressing slightly different data viewpoints and are consequently not fully compatible with each other. The fragmentation of data format specifications makes it hard to integrate and interpret data for further analysis with information from multiple data providers. As a solution, a new ontology is presented here for annotating and representing genomic feature and variation dataset contents. The Genomic Feature and Variation Ontology (GFVO) specifically addresses genomic data as it is regularly shared using the GFF3 (incl. FASTA), GTF, GVF and VCF file formats. GFVO simplifies data integration and enables linking of genomic annotations across datasets through common semantics of genomic types and relations.Availability and implementation: The latest stable release of the ontology is available via its base URI; previous and development versions are available at the ontology's GitHub repository: https://github.com/Biolnterchange/Ontologies ; versions of the ontology are indexed through BioPortal (without external class-/property-equivalences due to BioPortal release 4.10 limitations); examples and reference documentation is provided on a separate webpage: http://www.biointerchange.org/ontologies.html . GFVO version 1.0.2 is licensed under the CCO 1.0 Universal license ( https://creativecommons.org/publicdomain/zero/1.0) and therefore de facto within the public domain; the ontology can be appropriated without attribution for commercial and non-commercial use. 
Joachim Baran, Stanford Center for Biomedical Informatics Research, School of Medicine,

3 Stanford University, Stanford, CA 94305, USA*

140, Salt Lake City, UT 84108-3514, USA

City, UT 84108-3514, USA

\section{Høgskoleringen 5, N-7491 Trondheim, Norway}

Robert Hoehndorf, Computer, Electrical and Mathematical Sciences \& Engineering

Division and Computational Bioscience Research Center, King Abdullah University of Science and Technology, 4700 King Abdullah University of Science and Technology, Thuwal 23955-6900, Kingdom of Saudi Arabia

Michel Dumontier, Stanford Center for Biomedical Informatics Research, School of Medicine, Stanford University, Stanford, CA 94305, USA

* Corresponding author: kim@codamono.com

\section{Introduction}

As the cost of genomic laboratory experiments has fallen, vast amounts of genomic data are produced by high-throughput sequencing technologies as well as inexpensive microarrays in a wide range of studies (cf. Grada \& Weinbrecht, 2013; Guarnaccia et al., 2014). Large cancer genome repositories, e.g. the 12,807 cancer genomes sequenced by institutes of the International Cancer Genome Consortium (ICGC, release 18, http://www.icgc.org), contain extensive data about structural variations of DNA and proteins. Epigenetic cohort studies contribute substantially to the scientific knowledge base too, with millions of data points being provided by recent studies (Walker et al., 2011; Lam et al., 2012). Whilst the produced data are rich in information content, they are to varying degree kept in data silos due to the lack of a single unified standard for data exchange and data integration. This increases the cost of further data analysis, leaving much of that data not adequately used or simply unused (Sboner et al., 2011).

Genomic data is at the moment predominantly represented as plain text- or binary-data. Nucleotide or peptide sequences are expressible via the broadly known FASTA file format (Pearson and Lipman, 1988; included in the GFF3 format), sequencing alignments/maps can be encoded as SAM/BAM files (Li et al., 2009), genomic features of many kinds can be captured via GFF3 
33 (http://www.sequenceontology.org/gff3.shtml) or its predecessor GTF

34 (http://mblab.wustl.edu/GTF2.html); structural variations of DNA can be captured using VCF

35 (Danecek et al., 2011) or by the extended GFF3 file format GVF (Reese et al., 2010). Each of these

36 standards is specified using manifests written in plain English, and thus, by and large are neither

machine-interpretable - as opposed to merely machine-readable — nor formally precise, which leaves room for speculation about the encoding/representation of some data.

Markup-related file formats, such as the eXtensible Markup Language (XML;

http://www.w3.org/TR/2006/REC-xml11-20060816), provide a mitigated approach that maintains a

41 human-readable text file format, whilst at the same time offering rudimentary support for a formal data

42 format specification via Document Type Definitions or XML Schema (for a comparison between the 43 two standards see Bex et al., 2004). The Systems Biology Markup Language (SBML), (Hucka et al.,

44 2003), is an example of an XML-based format for representing computational models and biochemical networks. A similar approach towards representing protein-protein interaction networks culminated in

46 the data representation Proteomics Standards Initiative Molecular Interaction XML format (PSI-MI), 47 using an XML Schema for data validation (Hermjakob et al., 2004). A comparison among SBML, PSI48 MI and the BioPAX language, (Strömbäck and Lambrix, 2005), highlighted the advantages of 49 BioPAX's approach regarding data integration and linked data. BioPAX, (Demir et al., 2010), is a 50 specification formalized in the Web Ontology Language (OWL; Antoniou \& Harmelen, 2009), which

51 facilitates data integration and data linkage using the Resource Description Framework (RDF;

52 http://www.w3.org/TR/2014/REC-rdf11-concepts-20140225).

53 Some benefits of the Semantic Web technologies, such as RDF and OWL, have also been

54 highlighted in other fields. The Semantic Web has been suggested to enrich academic publications with 55 software interpretable annotations, (Shotton et al., 2009), a semantic linked data network of drug 56 targets has been presented as a data integration solution of heterogeneous drug related data sets in 57 (Chen et al., 2012), and a broader discussion on the role of the Semantic Web technologies in life 58 sciences was given in (Antezana et al., 2009).

\section{Generic Feature and Genomic Variation File Formats}

60 GFF3 is a cornerstone in many GMOD tools, (http://www.gmod.org), such as the generic 61 genome browser GBrowse (Stein et al., 2002), it is input to genomic variant annotation tools such as 62 ANNOVAR, (Wang et al., 2010), and it is used to format results of genome annotation pipelines such 
63 as MAKER, (Cantarel et al., 2008). The GFF3 specification also includes the FASTA file format 64 specification, since FASTA file contents are permitted in the trailing part of GFF3 files. Genomic 65 variations are represented using either GVF or VCF file formats, which are utilized interchangeably in 66 projects of the European Bioinformatics Institute's (EBI; Lappalainen et al., 2013), the 1000 Genomes

67 Project as well as the Broad Institute (The 1000 Genomes Project Consortium, 2012), among other data 68 providers.

GFF3-, GTF-, GVF-, and VCF-specifications define tab-delimited text file formats, which are instances of flat file formats. For all specifications, the text file contents are of variable column-length and additional predefined delimiters (such as a semicolon) are being used for further value separation within certain columns. Multiple Perl-inspired " 1 pragma" statements are typically declared in the beginning of the files to further determine the interpretation of genomic data contents below. Genomic data are either annotated in predefined columns (tab-delimited; column position determines data content), where some columns are allowed to contain multiple tag/value pairs, or, other composite or ordered data structures (e.g., lists). Figure 1 shows an example of a GFF3 file and the implicit relationships between data within it.

\section{From Flat Files to a Machine Interpretable Data Format}

Differences in the genomic feature and variation file formats require multiple software implementations for reading their data files, where data integration across file formats is difficult due to disparities in data labeling and data encoding. The goal of this paper is to overcome these issues by introducing the Genomic Feature and Variation Ontology (GFVO), which addresses this status quo by providing well described named ontological concepts and relations, by defining formal constraints on them, and by potentially enabling unified data access through a generic data representation.

GFVO predominantly unifies naming conventions and data encoding by introducing unique concepts for genomic features and variation representation. For example, the GFVO class "SequenceVariant" expresses sequence variants that are encoded using the "Variant_seq" attribute in GVF files and as either "ALT" column or "ALT" information field in VCF files. GFVO also resolves encoding differences between genomic feature and variation file formats. For example, gametic phase

is encoded very differently in GVF and VCF file formats. GVF denotes gametic phase with its

"Genotype" attribute in conjunction with "Phased" attribute, or alternatively, "\#\#phased-genotypes"

\footnotetext{
${ }^{1}$ http://perldoc.perl.org/index-pragmas.html
} 
92 pragma statement. VCF denotes gametic phase in its "GT" additional information field using the

93 special characters "|" (phased) and "”" (unphased). In GFVO, gametic phase is represented via the

94 unique class "GameticPhase" that encodes both GVF and VCF use cases.

95 Secondarily, GFVO enables easier data processing and data integration due to the use of World

96 Wide Web Consortium (W3C) backed data standards. Data represented by GFVO can be expressed in

97 one of the many interchangeable Semantic Web file formats (s.a. RDF N-Triples, RDF Turtle, or

98 JSON-LD). Data in those formats can be parsed in many popular programming languages, or, it can be

99 directly loaded into database management systems without requiring further data transformation. For

100 example, RDF Turtle can directly be imported into Virtuoso and other triple stores, while the newer

101 JSON-LD can be imported into MongoDB, Elasticsearch, and other NoSQL database management 102 systems.

\section{Methods}

104 GFVO is modeled in OWL and made available in RDF/XML format. ${ }^{2}$ The ontology's

105 expressiveness is within SRIQ with datatype properties, which is a Description Logic (Baader et al.,

106 2003) fragment whose development has previously been motivated by application in medical

107 terminology (Horrocks et al., 2005). Table 1 gives an overview of the number of classes, properties,

108 link-outs, and restrictions that are defined in GFVO.

\section{Ontology Design}

110 GFVO was designed to mirror the class and object property hierarchies of the Semanticscience

111 Integrated Ontology (SIO; Dumontier et al., 2014) release version 1.0.10. SIO is an ontology of basic

112 types and relationships for biomedical knowledge discovery that serves as an upper-level ontology to

113 GFVO. The term upper-level ontology has been defined as a highly abstracted ontology that does not

114 refer to identifiable and/or concrete entities of a domain, but also as an ontology that can be used to

115 impose structure on a lower-level ontology as well as provide relations to other ontologies (Musen,

116 2013). SIO adheres to the latter by ensuring semantic interoperability via relations between SIO classes

117 as well as properties and the Basic Formal Ontology (BFO; Grenon et al., 2004), Relation Ontology

118 (RO; Smith et al., 2005), and the Phenotypic Quality Ontology (PATO). ${ }^{3}$ Formal relationships between

${ }^{2}$ http://www.w3.org/TR/rdf-syntax-grammar

3 http://obofoundry.org/wiki/index.php/PATO:Main Page 
119 GFVO's and SIO's classes and properties are included in GFVO via "owl:equivalentProperty" and 120 "owl:equivalentClass" OWL-properties respectively.

121 Terminological requirements to describe data contents as covered by the GFF3 version 1.21, 122 GTF version 2, GVF version 1.07, and VCF version $4.1 \& 4.2$ specifications were gathered by 123 tabulating all named data elements within the specifications. Terminology from specifications was 124 preserved wherever possible, unless a single unifying term seemed more appropriate. This design 125 decision results in pre-coordinated GFVO classes. Pre-coordinated classes are understood as the combination of atomic concepts, e.g. GO accession "GO:0006260" that is labeled as "DNA replication", (Ashburner et al., 2000), whereas post-coordinated classes are interpreted as the combination of atomic classes via relations and axioms. The widely utilized SNOMED CT uses precoordinated terminology too, where post-coordination has been shown to have possible negative effects in terms of misinterpretation of composed SNOMED CT classes (Rector et al., 2012). Pre- and postcoordination have been studied in biomedical sciences in general and it has been found that precoordinated ontologies require less user guidance (Schulz et al., 2010). Table 2 provides an overview of the number of GFVO classes that are representing data as it can be found in the genomics feature and variation file formats.

Naming conventions in GFVO are requiring camel case rules for all identifiers, which have to be accompanied by an English-language label for classes and properties ("rdfs:label"). The camel case for class identifiers starts with an upper case letter (e.g., identifier "CodingFrameOffset" with "Coding Frame Offset" as "rdfs:label"). Object- and datatype-properties start with a lower case letter (e.g., identifier "isPartOf" with "is part of" as "rdfs:label"). Identifiers and labels in GFVO were chosen as close as possible to the genomics file format specifications to assist with the adoption of the ontology 141 by bioinformaticians and life science researches.

142 Similar to SIO, only one datatype property was modeled for representing literal values. Domain143 and range-restrictions have been placed as machine interpretable reasoning support that also serve as 144 application guide. Designated object properties have defined range restrictions to the Sequence 145 Ontology (SO; Eilbeck et al., 2005), Variation Ontology (VariO; Vihinen, 2013) and Feature 146 Annotation Location Description Ontology (FALDO; Bolleman et al., 2014, pre-print). The utilization 147 of existing ontologies for specific data representation needs facilitates data integration among genomic 148 data resources through ontology reuse. Examples are provided as Supplementary Information that show 
149 the intended use of GFVO including the use of the aforementioned ontologies in conjunction with 150 GFVO.

151 Descriptive explanations about GFVO's 102 classes, 33 properties and their intended usage have 152 been provided in comment sections ("rdfs:comment"; cf. tutorial by Horridge et al., 2007). It is shown

153 in Table 3 that the comment sections in GFVO add substantial documentation in comparison to 154 FALDO, SIO, SO, and VariO. Table 4 and Table 5 in the Supplementary Information break down 155 detailed mappings between the genomics specifications and GFVO, FALDO, SO, and RDF Schema; 156 Table 4 maps every GFVO class to data content in the genomic file formats, whereas Table 5 shows 157 use of FALDO, SO, and RDF Schema. Where applicable, GFVO references Wikipedia

158 ("rdfs:seeAlso") to provide extra information about the concept that classes encapsulate. References to 159 GFF3, GTF, GVF, and VCF specifications ("rdfs:isDefinedBy") are denoting the modeling origin as 160 well as applicability of classes to the respective file formats; ${ }^{4}$ the instantiation of classes is not 161 restricted by this annotation though and it is encouraged to make use of GFVO classes according to 162 data representation needs.

\section{Ontology Testing}

Protégé 4 $^{4} 3$ with HermiT 1.3.7 reasoner plug-in were used to test whether GFVO is free of inconsistencies and unsatisfiable classes - within the reasoning boundaries of HermiT. No

166 inconsistencies were highlighted by the reasoner and inferred class and property hierarchies have been manually evaluated and it has been determined that they follow the intended design.

168 Examples were created for selected use cases, which also serve as test bed for verifying the 169 ontology's expressiveness. Examples 1, 2, 3, 3a, 4, and 5 in the Supplementary Information show a 170 possible solution for encoding genes and their transcription factor binding sites, sequence alignments,

171 phased and unphased genotypes, sequence variants, and Phred quality scores, and data

172 filtering/annotating, respectively. All examples were inspected for errors using Protege/HermiT; no 173 errors were shown regarding the RDF Turtle format and the example are inconsistency free.

\section{Results}

4 The VCF specification is no longer maintained by the 1000 Genomes Project, but instead is maintained by the Global Alliance for Genomics and Health Data Working group file format team now. For backwards compatibility, GFVO includes links to the old as well as new location of the VCF 4.1 specification; the ontology also includes links to the current location of the VCF 4.2 specification. ${ }^{5}$ http://protege.stanford.edu 
GFVO unifies the vocabulary of the genomic feature and variation file format specifications by providing a well-defined and documented set of classes and properties that capture the gist of genomic data. Designated classes classes (e.g., "AlleleCount", “Zygosity”, "FragmentReadPlatform”) are provided for concepts that are only indicated by special and varied encodings in the file format specifications. Character based data encoding of the genomic feature and variation file formats, s.a. "|" for phased genotypes in the VCF specification, have been replaced by declarative classes with extensive documentation in GFVO.

Representing four genomic feature and variation file formats using GFVO enables easier access to the understanding and interpretation of genomic data. Independent of the file format specific encoding and formatting of the GFF3 (incl. FASTA), GTF, GVF, and VCF file formats, data represented using GFVO provides a single point of access for data interpretation due to its welldocumented class hierarchy. Genomic data represented using GFVO is also machine interpretable due to the adherence of Semantic Web standards as defined by the W3C.

\section{Discussion}

This work identified and formalized classes and relations in the genomics feature and variation file formats GFF3, GTF, GVF, and VCF. GFVO consolidates encodings across these file formats with distinct and machine interpretable concepts. It has been demonstrated by examples that GFVO is suitable for describing the data contents of common genomic feature and genomic variant file formats. Encoding genomic features and variations using GFVO comes at the cost of increased data size. This is partially due to the descriptive names that are featured in GFVO. For example, the representation of VCF allele counts (“AC" additional information) via the GFVO class "AlleleCount". Exclusive use of classes to represent genomic data is another contributing factor towards size, since each genomic datum needs to be encoded as a class instance. Use of multiple datatype properties could alleviate the size increase, but this was not implemented to stay in line with SIO design decisions. Size comparisons between GFF3-, GTF-, GVF-, and VCF-files and their Semantic Web representation fall beyond the scope of this paper; size requirements for Semantic Web file formats vary greatly between standards, some of which permit omission of repetitive information (s.a. RDF Turtle and JSON-LD) whilst other standards require the explicit encoding of all data (s.a. RDF N-Triples).

The ontology's distribution under the CC0 1.0 Universal license allows for adoption in commercial and non-commercial projects without the need to cite, to attribute, or otherwise reference 
206 the ontology, this paper, or its licensing terms in any form. GFVO is therefore de-facto within the 207 public domain.

\section{Conclusion}

An ontology - GFVO - for describing the file contents of GFF3, GTF, GVF, and VCF files was introduced. GFVO builds on the existing ontologies FALDO, SIO, and SO. It was demonstrated

211 that the ontology captures a set of use cases for describing genomic data; supporting evidence was

212 given that indicates that GFVO captures data of the genomics feature and variation formats completely.

\section{Acknowledgements}

214 Paul Avillach reviewed and provided feedback on an earlier version of the manuscript that 215 presented two ontologies specific to the GFF3 and GVF file formats; Chris Mungall provided feedback 216 regarding the GFF3 specific ontology as well. Raoul Jean Pierre Bonnal, Toshiaki Katayama,

217 Francesco Strozzi brought forward suggestions to improve GFVO's practical applications. Takatomo

218 Fujisawa corrected bugs in the late development stages of GFVO.

219 The organizers of BioHackathon and their supporting bodies - the Integrated Database Project

220 (Ministry of Education, Culture, Sports, Science and Technology of Japan), the National Bioscience

221 Database Center (NBDC), and the Database Center for Life Science (DBCLS) - enabled the

222 conception of the ontology.

\section{References}

224 The 1000 Genomes Project Consortium (2012). An integrated map of genetic variation from

225 1,092 human genomes. Nature, 491(7422), 56-65. doi:10.1038/nature11632

226 Antezana, E., Kuiper, M., Mironov, V. (2009). Biological knowledge management: the emerging 227 role of the Semantic Web technologies. Briefings in Bioinformatics, 10(4), pp. 392-407.

228 doi:10.1093/bib/bbp024

Antoniou, G., Harmelen, F. (2009). Web Ontology Language: OWL. Handbook on Ontologies SE - 4, pp. 91-110. Springer. doi:10.1007/978-3-540-92673-3_4

Ashburner, M., Ball, C.A., Blake, J.A., Botstein, D., Butler, H., Cherry, J.M., Davis, A.P.,

233 S., Matese, J.C., Richardson, J.E., Ringwald, M., Rubin, G.M., Sherlock, G., The Gene Ontology

234 Consortium (2000). Gene Ontology: tool for the unification of biology. Nature Genetics, 25(1), 25-29.

235 doi:10.1038/75556.Gene 
Baader, F., Calvanese, D., McGuinness, D. L., Nardi, D., Patel-Schneider, P. F. (Eds.). (2003).

The Description Logic Handbook: Theory, Implementation, and Applications. New York, NY, USA: Cambridge University Press.

Bex, G. J., Neven, F., Den Bussche, J. (2004). DTDs versus XML schema: a practical study. Proceedings of the 7th International Workshop on the Web and Databases: colocated with ACM SIGMOD/PODS 2004, pp. 79-84. doi:10.1145/1017074.1017095

Bolleman, J., Mungall, C. J., Strozzi, F., Baran, J., Dumontier, M., Bonnal, R. J. P., Buels, R., Hoehndorf, R., Fujisawa, T., Katayama, T., Cock, P. J. A. (2014). FALDO: A semantic standard for describing the location of nucleotide and protein feature annotation. bioRxiv.

Cantarel, B. L., Korf, I., Robb, S. M. C., Parra, G., Ross, E., Moore, B., Holt, C., Sánchez Alvarado, A., Yandell, M. (2008). MAKER: an easy-to-use annotation pipeline designed for emerging model organism genomes. Genome Research, 18(1), pp. 188-96. doi:10.1101/gr.6743907

Chen, B., Ding, Y., Wild, D. J. (2012). Assessing drug target association using semantic linked data. PLOS Computational Biology, 8(7). doi:10.1371/journal.pcbi.1002574

Danecek, P., Auton, A., Abecasis, G., Albers, C. A., Banks, E., DePristo, M. A., Handsaker, R. E., Lunter, G., Marth, G. T., Sherry, S. T., McVean, G., Durbin, R. (2011). The variant call format and VCFtools. Bioinformatics, 27(15), pp. 2156-8. doi:10.1093/bioinformatics/btr330

Demir, E., Cary, M. P., Paley, S., Fukuda, K., Lemer, C., Vastrik, I., Wu, G., D’Eustachio, P., Schaefer, C., Luciano, J., Schacherer, F., Martinez-Flores, I., Zhenjun, H., Jimenez-Jacinto, V., JoshiTope, G., Kandasamy, K., Lopez-Fuentes, A. C., Mi, H., Pichler, E., Rodchenkov, I., Splendani, A., Tkachev, S., Zucker, J., Gopinath, G., Rajasimha, H., Ramakrishnan, R., Shah, I., Syed, M., Anwar, N., Babur, Ö., Blinov, M., Brauner, E., Corwin, D., Donaldson, S., Gibbons, F., Goldberg, R., Hornbeck, P., Luna, A., Murray-Rust, P., Neumann, E., Ruebenacker, O., Samwald, M., van Iersel, M., Wimalaratne, S., Allen, K., Braun, B., Whirl-Carrillo, M., Cheung, K.-H., Dahlquist, K., Finney, A., Gillespie, M., Glass, E., Gong, L., Haw, R., Honig, M., Hubaut, O., Kane, D., Krupa, S., Kutmon, M., Leonard, J., Marks, D., Merberg, D., Petri, V., Pico, A., Ravenscroft, D., Ren, L., Shah, N., Sunshine, M., Tang, R., Whaley, R., Letovksy, S., Buetow, K. H., Rzhetsky, A., Schachter, V., Sobral, B. S., Dogrusoz, U., McWeeney, S., Aladjem, M., Birney, E., Collado-Vides, J., Goto, S., Hucka, M., Le Novère, N., Maltsev, N., Pandey, A., Thomas, P., Wingender, E., Karp, P. D., Sander, C., Bader, G. D. (2010). The BioPAX community standard for pathway data sharing. Nature Biotechnology, 28(9), pp. 935-42. doi:10.1038/nbt.1666 
R., Duck, G., Furlong, L. I., Keath, N., Klassen, D., McCusker, J. P., Queralt-Rosinach, N., Samwald, M., Villanueva-Rosales, N., Wilkinson, M. D., Hoehndorf, R. (2014). The Semanticscience Integrated Ontology (SIO) for biomedical research and knowledge discovery. Journal of Biomedical Semantics, 5(1), 14. doi:10.1186/2041-1480-5-14

Eilbeck, K., Lewis, S., Mungall, C., Yandell, M., Stein, L., Durbin, R., Ashburner, M. (2005).

The Sequence Ontology: a tool for the unification of genome annotations. Genome Biology, 6(5), R44. doi:10.1186/gb-2005-6-5-r44

Grada, A., Weinbrecht, K. (2013). Next-generation sequencing: methodology and application. The Journal of Investigative Dermatology, 133(8), e11. doi:10.1038/jid.2013.248

Grenon, P., Smith, B., Goldberg, L. (2004). Biodynamic ontology: Applying BFO in the Biomedical Domain. Ontologies in Medicine, (ii), 20-38.

Guarnaccia, M., Gentile, G., Alessi, E., Schneider, C., Petralia, S., Cavallaro, S. (2014). Is this the real time for genomics? Genomics, 103(2-3), 177-82. doi:10.1016/j.ygeno.2014.02.003 Orchard, S., Sarkans, U., von Mering, C., Roechert, B., Poux, S., Jung, E., Mersch, H., Kersey, P., Lappe, M., Li, Y., Zeng, R., Rana, D., Nikolski, M., Husi, H., Brun, C., Shanker, K., Grant, S. G. N., Sander, C., Bork, P., Zhu, W., Pandey, A., Brazma, A., Jacq, B., Vidal, M., Sherman, D., Legrain, P., Cesareni, G., Xenarios, I., Eisenberg, D., Steipe, B., Hogue, C., Apweiler, R. (2004). The HUPO PSI's Molecular Interaction format - a community standard for the representation of protein interaction data. Nature Biotechnology, 22(2), pp. 177-183. doi:10.1038/nbt926

Horridge, M., Jupp, S., Moulton, G., Rector, A., Stevens, R., Wroe, C. (2007). A Practical Guide To Building OWL Ontologies Using The Protege-OWL Plugin and CO-ODE Tools Edition 1.1. University of Manchester, pp. 1-102. Retrieved from

\section{2 (accessed February 8, 2013)}

Horrocks, I., Kutz, O., Sattler, U. (2005). The Irresistible SRIQ. In In Proceedings of OWL:

294 Experiences and Directions.

Hucka, M., Finney, A., Sauro, H. M., Bolouri, H., Doyle, J. C., Kitano, H., Arkin, A. P., 297 Gor, V., Goryanin, I. I., Hedley, W. J., Hodgman, T. C., Hofmeyr, J.-H., Hunter, P. J., Juty, N. S., 
298 Kasberger, J. L., Kremling, A., Kummer, U., Le Novère, N., Loew, L. M., Lucio, D., Mendes, P., 299 Minch, E., Mjolsness, E. D., Nakayama, Y., Nelson, M. R., Nielsen, P. F., Sakurada, T., Schaff, J. C., 300 Shapiro, B. E., Shimizu, T. S., Spence, H. D., Stelling, J., Takahashi, K., Tomita, M., Wagner, J., 301 Wang, J. (2003). The systems biology markup language (SBML): a medium for representation and 302 exchange of biochemical network models. Bioinformatics, 19(4), pp. 524-531.

303 doi:10.1093/bioinformatics/btg015

304 Lam, L. L., Emberly, E., Fraser, H. B., Neumann, S. M., Chen, E., Miller, G. E., Kobor, M. S. 305 (2012). Factors underlying variable DNA methylation in a human community cohort. Proceedings of 306 the National Academy of Sciences of the United States of America, 109(2), pp. 17253-60.

307 doi:10.1073/pnas.1121249109

308 Lappalainen, I., Lopez, J., Skipper, L., Hefferon, T., Spalding, J. D., Garner, J., Chen, C., 309 Maquire, M., Corbett, M., Zhou, G., Paschall, J., Ananiev, V., Flicek, P., Church, D. M. (2013). DbVar 310 and DGVa: public archives for genomic structural variation. Nucleic Acids Research, 41(Database 311 issue), D936-41. doi:10.1093/nar/gks1213

312 Li, H., Handsaker, B., Wysoker, A., Fennell, T., Ruan, J., Homer, N., Marth, G., Abecasis, G., 313 Durbin, R., 1000 Genome Project Data Processing Subgroup (2009). The Sequence Alignment/Map 314 format and SAMtools. Bioinformatics, 25(16), pp. 2078-9. doi:10.1093/bioinformatics/btp352

Musen, M. (2013). Upper Level Ontology. In W. Dubitzky, O. Wolkenhauer, K.H. Cho, H. Yokota (Eds.), Encyclopedia of Systems Biology SE - 1520 (p. 2328). Springer New York. doi:10.1007/978-1-4419-9863-7_1520

Pearson, W. R., Lipman, D. J. (1988). Improved tools for biological sequence comparison. Proceedings of the National Academy of Sciences of the United States of America, 85(8), pp. $2444-8$. doi:10.1073/pnas.85.8.2444

Rector, A., Iannone, L. (2012). Lexically suggest, logically define: quality assurance of the use of qualifiers and expected results of post-coordination in SNOMED CT. Journal of Biomedical Informatics, 45(2), 199-209. doi:10.1016/j.jbi.2011.10.002

Reese, M. G., Moore, B., Batchelor, C., Salas, F., Cunningham, F., Marth, G. T., Stein, L., Flicek, P., Yandell, M., Eilbeck, K. (2010). A standard variation file format for human genome sequences. Genome Biology, 11(8). doi:10.1186/gb-2010-11-8-r88

Sboner, A., Mu, X., Greenbaum, D., Auerbach, R., Gerstein, M. (2011). The real cost of sequencing: higher than you think! Genome Biology, 12(8). 
Schulz, S., Schober, D., Raufie, D., Boeker, M. (2010). Pre-and postcoordination in biomedical 330 ontologies. In Herre, H., Hoehndorf, R., Kelso, J., Schulz, S. (Eds.), OBML 2010 Workshop (Vol. 6).

331 Retrieved from http://www.onto-med.de/obml/ws2010/obml2010report.pdf\#page=65 (accessed

332 December 9, 2014)

333 Shotton, D., Portwin, K., Klyne, G., Miles, A. (2009). Adventures in semantic publishing:

334 exemplar semantic enhancements of a research article. PLOS Computational Biology, 5(4).

335 doi:10.1371/journal.pcbi.1000361

336 Smith, B., Ceusters, W., Klagges, B., Köhler, J., Kumar, A., Lomax, J., Mungall, C., Neuhaus,

337 F., Rector, A.L., Rosse, C. (2005). Relations in biomedical ontologies. Genome Biology, 6(5), R46.

338 doi:10.1186/gb-2005-6-5-r46

339 Stein, L. D., Mungall, C., Shu, S., Caudy, M., Mangone, M., Day, A., Nickerson, E., Stajich, J.

340 E., Harris, T. W., Arva, A., Lewis, S. (2002). The generic genome browser: a building block for a

341 model organism system database. Genome research, 12(10), 1599-610. doi:10.1101/gr.403602

342 Strömbäck, L., Lambrix, P. (2005). Representations of molecular pathways: an evaluation of

343 SBML, PSI MI and BioPAX. Bioinformatics, 21(24), pp. 4401-7. doi:10.1093/bioinformatics/bti718

344 Vihinen, M. (2013). Variation Ontology for annotation of variation effects and mechanisms.

345 Genome Research. doi:10.1101/gr.157495.113

346 Walker, B. a, Wardell, C. P., Chiecchio, L., Smith, E. M., Boyd, K. D., Neri, A., Davies, F. E.,

347 Ross, F. M., Morgan, G. J. (2011). Aberrant global methylation patterns affect the molecular

348 pathogenesis and prognosis of multiple myeloma. Blood, 117(2), pp. 553-62. doi:10.1182/blood-2010-

$349 \quad 04-279539$

350 Wang, K., Li, M., Hakonarson, H. (2010). ANNOVAR: functional annotation of genetic variants

351 from high-throughput sequencing data. Nucleic Acids Research, 38(16). doi:10.1093/nar/gkq603 


\section{Table $\mathbf{1}$ (on next page)}

Overview of classes and properties in GFVO.

Total number of classes and properties in GFVO, number of classes/properties that have equivalences to SIO, number of classes based on GFF3, GTF, GVF and VCF specifications (not mutually exclusive), number of classes with reference to Wikipedia articles, disjointness axioms, and property restrictions. 


\begin{tabular}{|c|c|c|c|}
\hline & Total Number & Equivalence with SIO & Equivalence with SO \\
\hline Classes & 102 & 40 & 13 \\
\hline ...modeled from GFF3 & 23 & 11 & 3 \\
\hline ...modeled from GTF & 13 & 11 & 2 \\
\hline ...modeled from GVF & 62 & 23 & 12 \\
\hline ...modeled from VCF & 42 & 15 & 7 \\
\hline \multicolumn{4}{|l|}{ Class Metadata } \\
\hline ...Wikipedia references & 53 & $\mathrm{n} / \mathrm{a}$ & $\mathrm{n} / \mathrm{a}$ \\
\hline ...pairwise disjoint axioms & 6 & $\mathrm{n} / \mathrm{a}$ & $\mathrm{n} / \mathrm{a}$ \\
\hline ...disjoint collection axioms & 13 & $\mathrm{n} / \mathrm{a}$ & $\mathrm{n} / \mathrm{a}$ \\
\hline ....with property restrictions & 32 & $\mathrm{n} / \mathrm{a}$ & $\mathrm{n} / \mathrm{a}$ \\
\hline Datatype Properties & 1 & 1 & 0 \\
\hline Object Properties & 32 & 31 & 0 \\
\hline
\end{tabular}


Table 2 (on next page)

Overview of file-format data structures captured by GFVO.

Overview of the number of data structures (columns, key/value pairs, other) in genomics file formats that are captured by GFVO and other ontologies/frameworks. 


\begin{tabular}{|l|l|l|l|}
\hline $\begin{array}{l}\text { Specificati } \\
\text { on }\end{array}$ & Fixed Columns & $\begin{array}{l}\text { Feature Attributes and } \\
\text { Key/Value Properties } \\
\text { Table } \\
\begin{array}{l}\text { Column } \\
\text { Description } \\
\text { keolean attributes and } \\
\text { associated with genomic } \\
\text { features }\end{array}\end{array}$ & $\begin{array}{l}\text { Pragma Statements and } \\
\text { Information Fields } \\
\text { typer information that is } \\
\text { stated in the header or } \\
\text { outside of the scope of } \\
\text { genomic feature annotations } \\
\text { (e.g., comments or FASTA } \\
\text { appendix) }\end{array}$ \\
\hline & $\begin{array}{l}\text { entities identified in specification and represented by classes in GFVO; entities represented } \\
\text { in other ontologies/frameworks as either class or property are noted in brackets }\end{array}$ \\
\hline GFF3 & $8(+1$ SO) & 5 & 5 (+4 RDF Schema) \\
\hline GTF & 9 & n/a & 6 \\
\hline GVF & $8(+1$ SO) & 25 & 27 (+4 RDF Schema) \\
\hline VCF & 6 & 24 & 15 \\
\hline
\end{tabular}




\section{Table 3(on next page)}

Coverage of class documentation in terms of word- and class-counts.

Coverage of class documentation in genomics related ontologies. Coverage is denoted by total number of words of documentation as well as on a normalized per-class basis. 


\begin{tabular}{|l|r|l|r|}
\hline Ontology & $\begin{array}{l}\text { Total Number of } \\
\text { Words in } \\
\text { Comments/Descripti } \\
\text { ons }\end{array}$ & $\begin{array}{l}\text { Total Number of } \\
\text { Classes }\end{array}$ & $\begin{array}{l}\text { Average Number of Words in } \\
\text { Descriptions per Class }\end{array}$ \\
$\begin{array}{l}\text { FALDO 31st November } \\
\text { 2014 }\end{array}$ & 477 & 18 & $\begin{array}{c}\text { average (minimum; median; } \\
\text { maximum) }\end{array}$ \\
\hline GFVO 1.0.2 & 4478 & 102 & $26.5(6 ; 25 ; 72)$ \\
\hline SIO 1.0.10 & 23412 & 1414 & $43.9(12 ; 38.5 ; 133)$ \\
\hline SO 1.329 & 7296 & 2254 & $16.56(5 ; 16 ; 73)$ \\
\hline $\begin{array}{l}\text { VariO 16th January } \\
\text { 2014 }\end{array}$ & 4612 & 384 & $3.24(1 ; 13 ; 97)$ \\
\hline
\end{tabular}


A GFF3 file example that shows the implicit relationships between data in the file and its genomic locus interpretation.

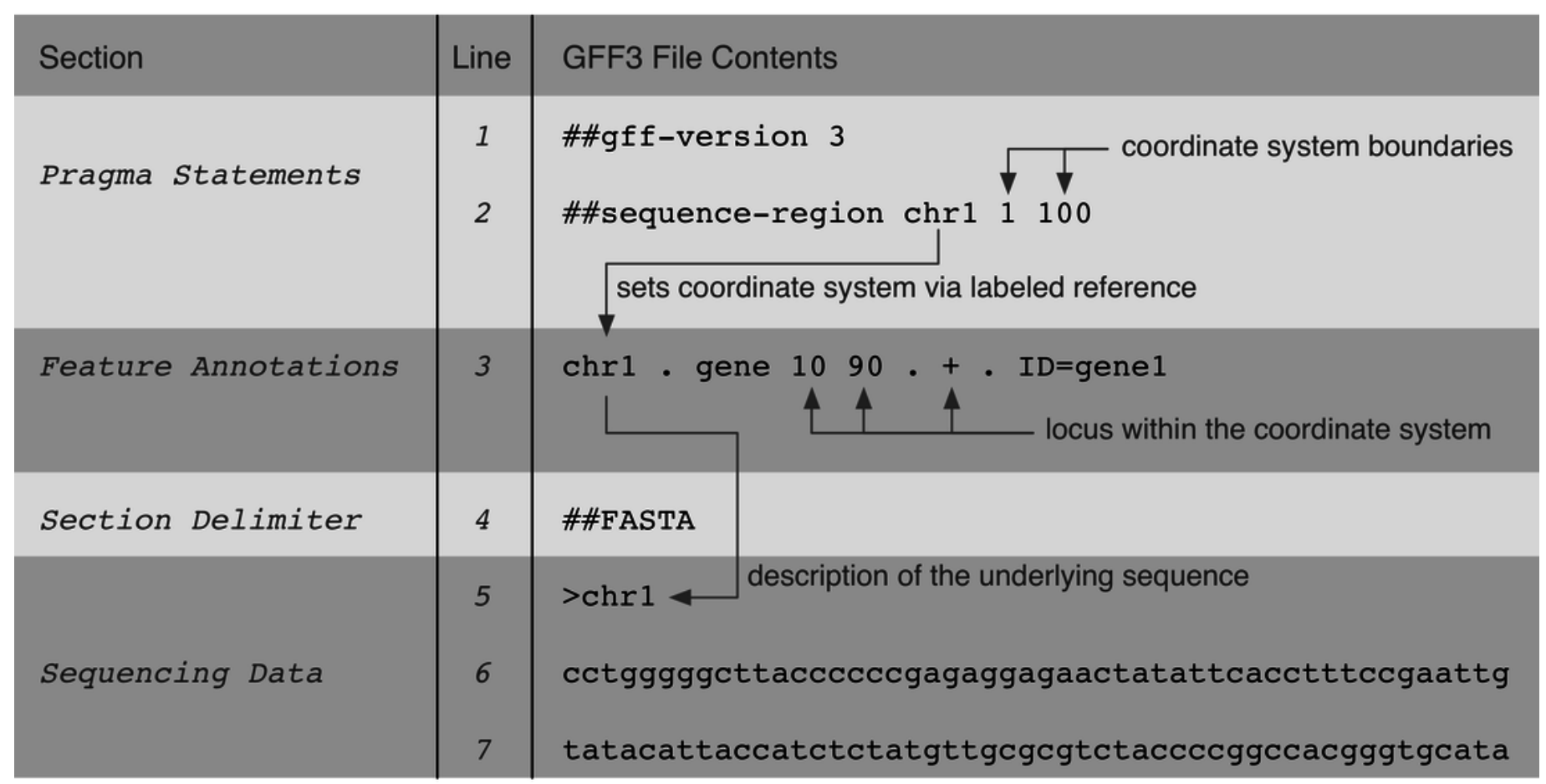

